\title{
Comparison of Magnetic Resonance Imaging Findings between Pathologically Proven Cases of Atypical Tubercular Spine and Tumour Metastasis: A Retrospective Study in 40 Patients
}

\author{
Somit Mittal ${ }^{1}$, Mohd Khalid ${ }^{1}$, Aamir Bin Sabir ${ }^{2}$, Saifullah Khalid ${ }^{1}$ \\ ${ }^{1}$ Department of Radiodiagnosis, Jawahar Lal Nehru Medical College, Aligarh Area, India \\ ${ }^{2}$ Department of Orthopaedics, Jawahar Lal Nehru Medical College, Aligarh Area, India
}

\begin{abstract}
Study Design: Retrospective study.
Purpose: To note the magnetic resonance imaging (MRI) differences between pathologically proven cases of atypical spinal tuberculosis and spinal metastasis in 40 cases.

Overview of Literature: Spinal tuberculosis, or Pott's spine, constitutes less than $1 \%$ of all cases of tuberculosis and can be associated with a neurologic deficit. Breast, prostate and lung cancer are responsible for more than $80 \%$ of metastatic bone disease cases, and spine is the most common site of bone metastasis. Thus, early diagnosis and prompt management of these pathologies are essential in preventing various complications.

Methods: We retrospectively reviewed 40 cases of atypical tuberculosis and metastasis affecting the spine from the year 2012 to 2014, with 20 cases each that were proven by histopathological examination. MR imaging was performed on 1.5 T MR-Scanner (Magnetom Avanto, Siemens) utilizing standard surface coils of spine with contrast injection. Chi-square test was used for determining the statistical significance and $p$-values were calculated.

Results: The most common site of involvement was the thoracic spine, seen in $85 \%$ cases of metastasis and $65 \%$ cases of Pott's spine $(p=0.144)$. The mean age of patients with tubercular spine was found to be 40 years and that of metastatic spine was 56 years. The following MR imaging findings showed statistical significance $(p<0.05)$ : combined vertebral body and posterior elements involvement, skip lesions, solitary lesion, intra-spinal lesions, concentric collapse, abscess formation and syrinx formation.

Conclusions: Tuberculosis should be considered in the differential diagnosis of various spinal lesions including metastasis, fungal spondylodiskitis, sarcoidosis and lymphoma, particularly in endemic countries. Spinal tuberculosis is considered one of the great mimickers of disease as it could present in a variety of typical and atypical patterns, so proper imaging must be performed in order to facilitate appropriate treatment.
\end{abstract}

Keywords: Spine; Infection; Tuberculosis; Metastasis; Magnetic resonance imaging

Received Nov 21, 2015; Revised Jan 15, 2016; Accepted Jan 19, 2016

Corresponding author: Somit Mittal

Department of Radiodiagnosis, Jawaharlal Nehru Medical College, Aligarh Muslim University,

Aligarh- 202001, Uttar Pradesh (UP), India

Tel: +91-9897900306, E-mail: somit999@gmail.com 


\section{Introduction}

Spinal tuberculosis, also known as Pott's disease of the spine, is one of the frequently encountered extra-pulmonary forms of the disease particularly in developing nations. For developed nations, the disease is usually associated with the migrants from the endemic countries. Despite its common occurrence and the high frequency of long-term morbidity, spinal tuberculosis is sometimes difficult to diagnose. In many cases, the clinical distinctions between Pott's disease and tumour metastasis are not typical, such as in central Pott's disease, which shows vertebral involvement without associated discal changes and neural arch tuberculosis. Biopsy is the ultimate tool for the final diagnosis; however, various patterns of involvement of the spine help in identification of the pathologies.

The purpose of this study was to determine the accuracy of magnetic resonance imaging (MRI) in differentiating the atypical cases of spinal tuberculosis and metastasis.

\section{Materials and Methods}

We retrospectively reviewed a total of 40 cases of atypical tuberculosis and metastasis affecting the spine from years 2012 to 2014. The mean age of patients with tubercular spine was less than or equal to 40 years (range, 11-67 years) and that for metastatic spine was less than or equal to 56 years (range, 43-70 years). All patients presented with back pain and weakness involving bilateral lower limbs in both symmetrical and asymmetrical fashion. Few patients were also having bladder and bowel dysfunction. The average duration of presentation of symptoms were around 5-6 months in tubercular spine and for 2-3 months in metastasis. There were 20 cases each of tubercular spine and metastasis, which were proven by histopathological examination. Out of 20 cases of metastasis spine, 7 cases were from lung primary, 6 from breast carcinoma, 1 from glottic carcinoma, 4 from prostate carcinoma and 2 from unknown primary tumours.

MR imaging was performed on a $1.5 \mathrm{~T}$ helium cooled superconducting MR scanner (Magnetom Avanto 1.5T, Siemens, Germany) utilizing standard surface coils for the cervical, lumbar and thoracic regions of spine. All the images were obtained using multi-slice spin echo sequences. Sagittal and coronal T1-weighted spin echo MR images with a short repetition time and a short echo time (350500/11-30), sagittal and axial T2-weighted turbo spin- echo image with long repetition time and long echo time $(3,000-7,000 / 120-150)$ and coronal short tau inversion recovery (STIR) (3,000-5,000/39-55). Other sequences like gradient echo sequence, etc. were obtained as and when required. Optimark (Gadoversetamide) contrast injection was administered at the dose of $0.1 \mathrm{mmol} / \mathrm{kg}$ and post contrast fat saturated axial and sagittal T1-weighted images (350-400/11-30) were obtained. Typical MR image acquisition parameters include slice thickness of 3 $\mathrm{mm}$, inter-slice gap of $1 \mathrm{~mm}$ and matrix size of $125 \times 308$. Before performing MR examination, details of the study were explained to the patient and a written consent was obtained.

Atypical spinal column involvement included atypical vertebral involvement without discal changes, vertebral plana, sclerotic vertebra, intra-spinal granuloma formation (epidural, intradural and intra-medullary) and epidural extra-osseous abscess formation. Vertebral involvement was considered when it showed high signal intensity on T2 and STIR images. When the adjacent vertebral bodies were involved without any involvement of IV disc space involvement, consecutive or skip pattern was considered. When only the single vertebral body was involved, solitary pattern was considered. The vertebral lesions were classified on the basis of anterior or posterior element involvement, both elements of involvement, and pattern of involvement, i.e. solitary or consecutive without intervertebral disc involvement.

Various imaging parameters were considered, which included presence or absence of abscess formation in paraspinal, intraosseous and epidural space, meningeal enhancement and syrinx formation. An abscess was defined as altered signal intensity lesion appearing hyper-intense on T2 and STIR with peripheral rim pattern of enhancement on post contrast scans.

\section{Results}

The distinction between spinal tuberculosis and metastasis could be made on MR imaging (Table 1). The most common site of involvement was the thoracic spine, seen in $85 \%(17 / 20)$ of metastasis cases and $65 \%(13 / 20)$ of Pott's spine cases $(p=0.144$ ), as shown in Table 2 . The various presentation of lesions included solitary lesion, concentric collapse type, intra-spinal type, leptomeningeal pattern of involvement and the vertebral body changes (anterior, neural arch and combined). These patterns of 
Table 1. MR findings of metastasis and atypical spinal tuberculosis

\begin{tabular}{lccc} 
MR findings & Metastasis $(n=20)$ & Tubercular spine $(n=20)$ & $p$-value ${ }^{\text {a) }}$ \\
\hline Most common site (thoracic region) & $85(17 / 20)$ & $5(1 / 20)$ & 0.144 \\
Isolated posterior elements involvement & $20(4 / 20)$ & $65(13 / 20)$ & 0.151 \\
\hline Isolated anterior element involvement & $55(11 / 20)$ & $70(14 / 20)$ & 0.520 \\
Combined elements involvement & $75(15 / 20)$ & $20(4 / 20)$ & 0.723 \\
\hline Skip lesions & $65(13 / 20)$ & $60(12 / 20)$ & 0.003 \\
Intra-spinal lesions (granulomas/metastasis) & $20(4 / 20)$ & $10(2 / 20)$ & 0.009 \\
\hline Concentric collapse & $60(12 / 20)$ & $5(1 / 20)$ & 0.000 \\
\hline Solitary lesion & $35(7 / 20)$ & & 0.017 \\
\hline Abscess & & $30(6 / 20)$ & 0.007 \\
\hline Para-spinal abscess & $0(0 / 20)$ & $25(5 / 20)$ & 0.016 \\
\hline Intra-osseous abscess & $0(0 / 20)$ & $55(11 / 20)$ & $<0.001$ \\
\hline Epidural abscess & $0(0 / 20)$ & $45(9 / 20)$ & 0.091 \\
\hline Meningeal enhancement & $20(4 / 20)$ & $40(8 / 20)$ & 0.030 \\
\hline Syrinx formation & $10(2 / 20)$ & & \\
\hline
\end{tabular}

Values are presented as \% (no. of cases).

$\mathrm{MR}$, magnetic resonance image.

${ }^{\text {a) }}$ hi-square test.

Table 2. Distribution of cases

\begin{tabular}{lcc} 
Location & Metastasis $(\mathrm{n}=20)$ & Tubercular Spine $(\mathrm{n}=20)$ \\
\hline Cervical & $1(5)$ & $2(10)$ \\
Upper thoracic & $4(20)$ & $3(15)$ \\
Lower thoracic & $13(65)$ & $10(50)$ \\
Lumbar & $1(5)$ & $3(15)$ \\
\hline Sacrum/coccyx & $1(5)$ & $2(10)$ \\
\hline
\end{tabular}

Values are presented as no. of patients (\%).

involvement have been discussed in the subsequent paragraphs.

Vertebral involvement has shown various key features helpful in differentiating the two aforementioned entities. Isolated anterior element (vertebral body) involvement was seen in $55 \%$ of metastasis cases and in $65 \%$ of Pott's spine cases. This pattern was not significant having a $p$-value of 0.520 . Isolated involvement of posterior elements (lamina, pedicles and spinous process) was more commonly seen in metastasis as compared to tubercular spine (20\% in metastasis vs. $5 \%$ in Pott's spine), however, this was not statistically significant with a $p$-value of 0.151 . Combined anterior and posterior elements involvement was seen more in the metastatic spine $(75 \%$ of metastasis vs. $70 \%$ of Pott's spine cases) (Figs. 1, 2). This was, how-

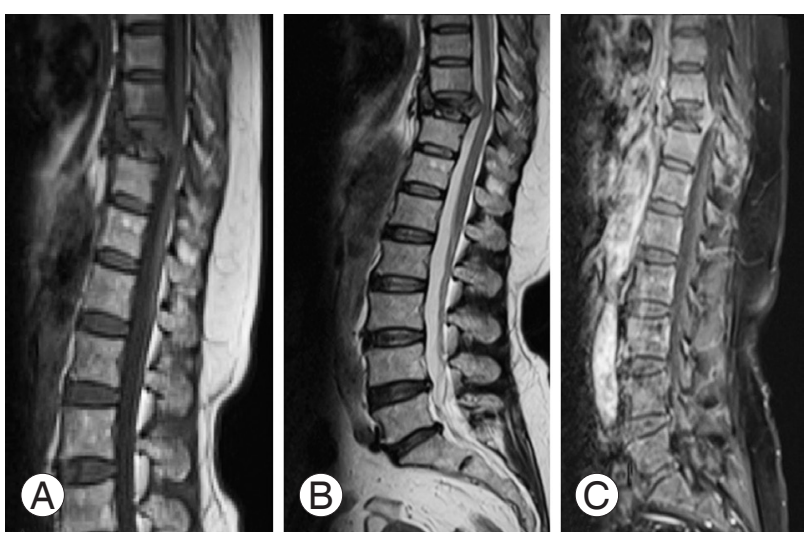

Fig. 1. Sagittal T1-weighted (A) and T2-weighted (B) images of dorsolumbar spine shows collapse of D10 vertebra with compression of thecal sac and maintained intervertebral disc space, which on post contrast T1-weighted fat sat image (C) show heterogeneous enhancement. Histo-pathological examination revealed metastatic adenocarcinoma. 


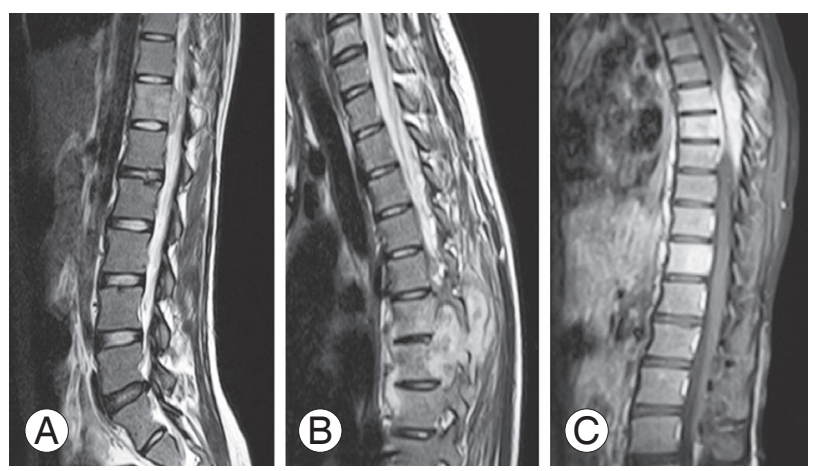

Fig. 2. Sagittal T2-weighted images of lumbo-sacral spine (A) and dorsolumba spine (B) show hyper-intense signal involving the D5, D6 and D12 vertebra with soft tissue component and sparing of IV discs which on post contrast scan (C) revealed heterogeneous enhancement in the body, posterior elements and epidural space with compressive myelopathy. Cytology revealed granuloma formation.
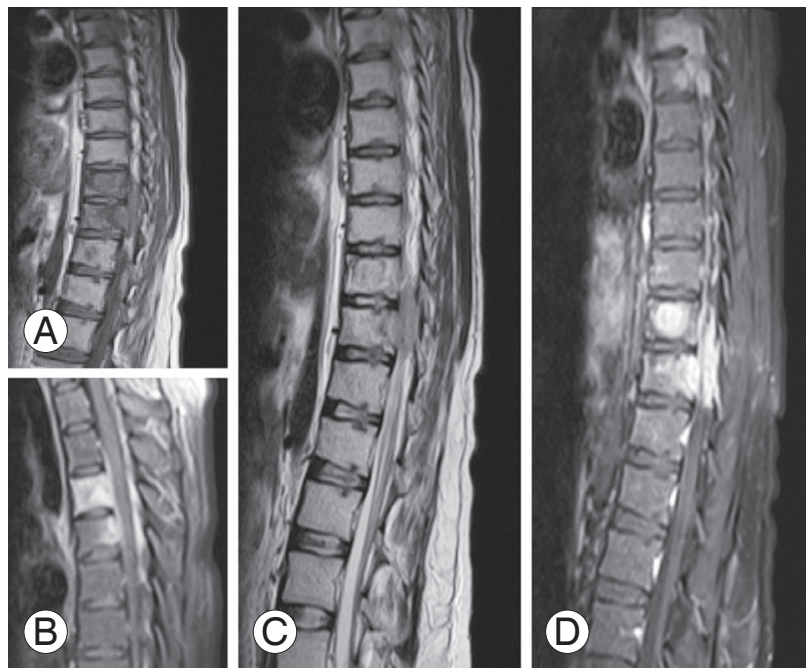

Fig. 3. Sagittal T1-weighted (A) and T2-weighted (C) images of dorsal spine show an altered signal intensity lesion involving the body and epidural space, which on post contrast scan (B, D) showed homogeneous pattern of enhancement along with lepto-meningeal extension. Cerebrospinal fluid analysis revealed increased lymphocytes which stained positive for acid-fast bacillus.

ever, not significant at $p<0.05$.

Skip lesions showed statistical significance $(p<0.05)$ seen in more cases of metastasis (65\% vs. $20 \%$ ) (Figs. 2, 3). The site of skip lesions showed involvement of thoracic spine in 10 cases for metastasis as compared to tuberculosis for which 6 and 4 cases involved thoracic and thoraco-lumbar spine, respectively. Remaining regions included the cervical, lumbar and sacrum of the spine. Solitary lesions of the spine were a feature seen in metastasis (35\%) as compared to Pott's spine (5\%), in which only 1 case showed a single

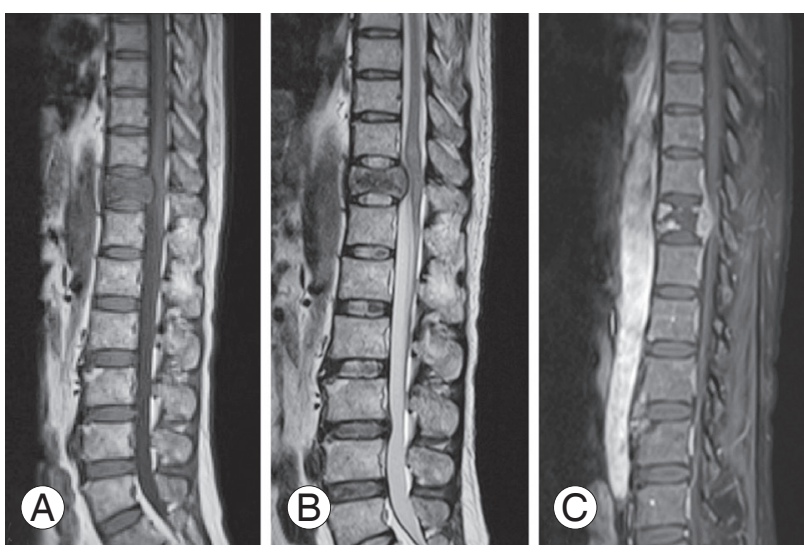

Fig. 4. Sagittal T1-weighted (A) and T2-weighted (B) images show fracture of D10 vertebra with posterior convexity and maintained adjoining IV disc space. On post contrast scan (C) there was noted heterogeneous pattern with the central part being necrotic. Histopathological examination revealed metastatic squamous cell carcinoma.
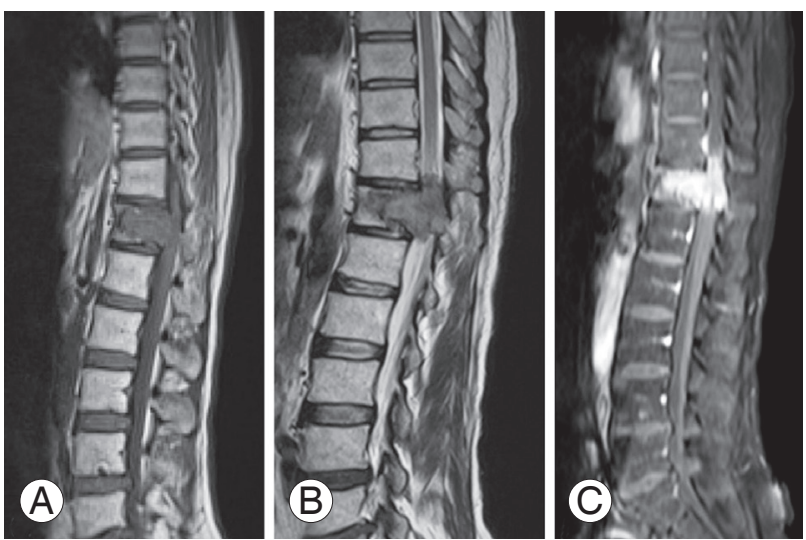

Fig. 5. Sagittal T1-weighted (A) and T2-weighted (B) images of dorsolumbar spine show altered signal intensity lesion involving the body of D11 vertebra with epidural extension leading to compressive myelopathy. On post contrast scan (C), the lesion showed heterogeneous enhancement.

lesion without intervertebral disc changes (Figs. 4, 5).

Intra-spinal tuberculoma formation was seen in $60 \%$ of the cases as compared to $20 \%$ of metastasis cases, which were mainly from breast carcinoma $(p<0.009)$ (Fig. 6). Concentric collapse was seen in $10 \%$ of Pott's spine cases versus $60 \%$ of metastasis cases $(p<0.001)$ (Figs. $1,7,8)$.

Of note, contrast enhancement pattern showed no statistical difference between them as only heterogeneous patterns were seen.

Abscess formation appeared hypo- to iso-intense on T1-weighted images and hyper-intense on T2-weighted images and STIR sequence with peripheral pattern of enhancement on contrast study. The abscess formation 
was seen in paravertebral, prevertebral, intra-osseous and epidural spaces (Figs. 2, 3, 9). This feature was solely restricted to the Pott's spine cases, which was statistically significant $(p<0.05)$.

The involvement of meninges was seen as a continuous enhancement along the spinal cord on post contrast scan (Figs. 3, 10). This was seen in $45 \%$ of Pott's spine cases and $20 \%$ of metastasis cases with $p$-value around 0.091 ,

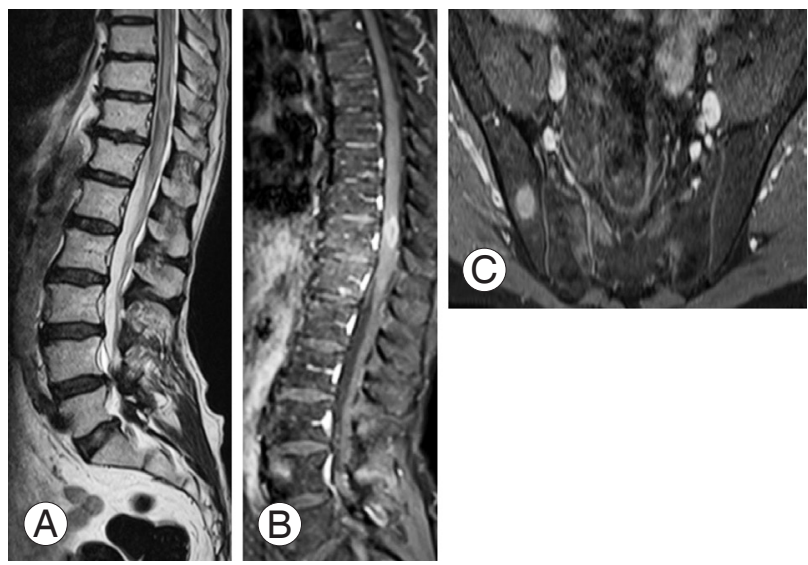

Fig. 6. Sagittal T2-weighted (A) image shows a round altered signal intensity, involving intramedullary region with syrinx formation, which on post contrast scan (B) shows ring enhancement. Additional finding noted was similar lesions in right sacro-iliac joint (C). The patient improved on antitubercular treatment. which was not significant. Syrinx formation was the other associated finding, observed more in Pott's spine (10\% of metastasis vs. $40 \%$ of Pott's spine cases), and was probably related to obstruction of the cerebrospinal fluid (CSF) $(p<0.05)$ (Fig. 10).
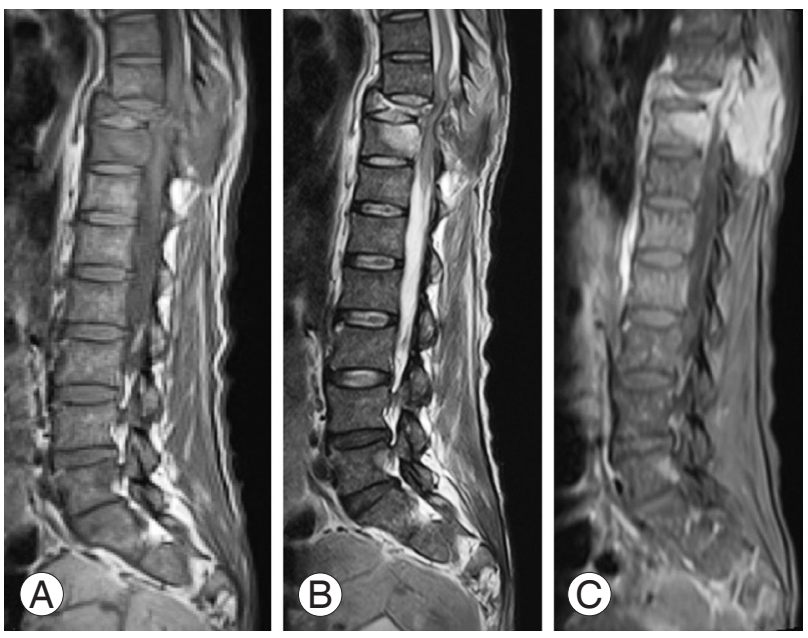

Fig. 7. Sagittal T1-weighted (A) and T2-weighted (B) images show collapsed D10 vertebra with retro-listhesis of D9 vertebra with compressive myelopathic changes and altered signal intensity in D10 and D11 body and posterior elements. On post contrast sagittal T1 fatsaturated image $(\mathbf{C})$, there is homogeneous pattern of enhancement involving the vertebras, epidural spaces and leptomeninges. Histopathological examination revealed granulomas.
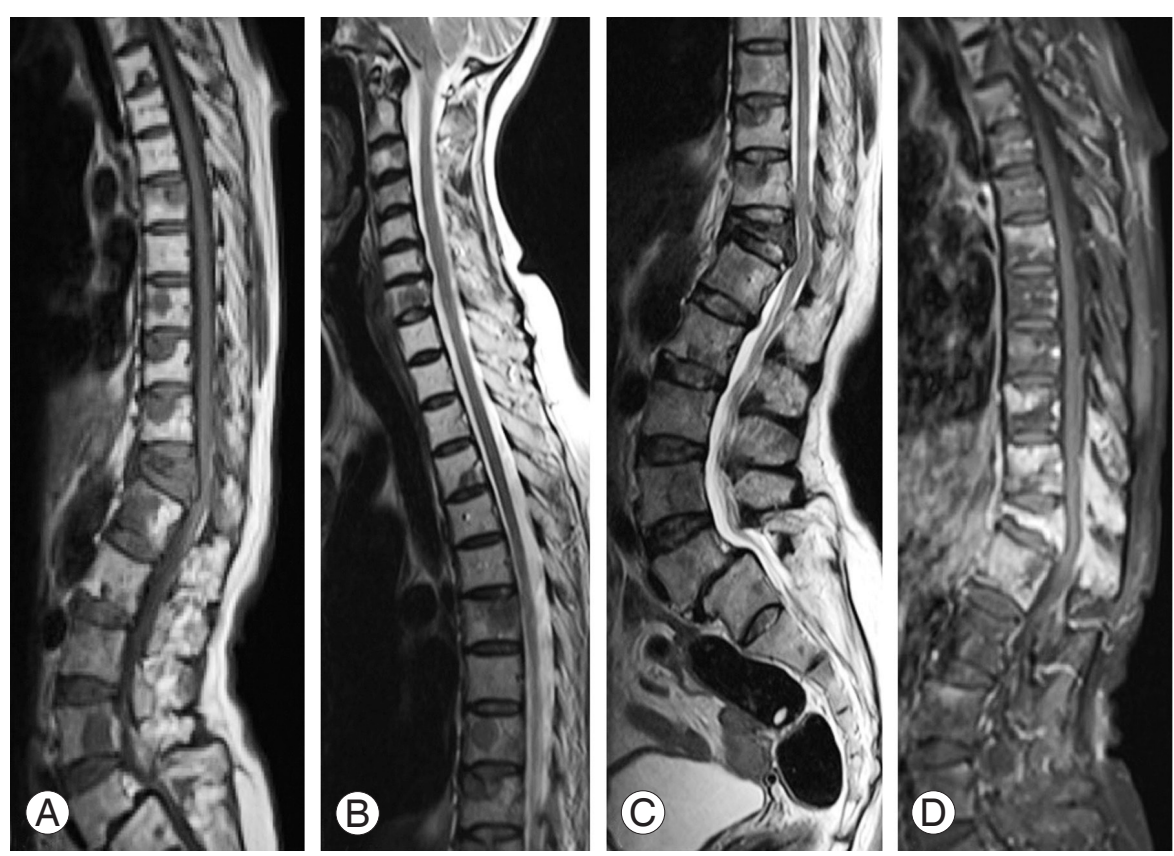

Fig. 8. Sagittal T1-weighted (A) and T2-weighted (B, C) images of dorsal and cervical spine show multiple areas of altered signal intensity involving almost all of the vertebras with compression fracture of D12 vertebra. Post contrast scan (D) shows heterogeneous patchy enhancement of these lesions. 
Findings like lung consolidation, pleural empyema, sacroilitis and communicating hydrocephalus were associated with tubercular spine only (Fig. 10). No association of these findings were noted for metastasis cases.

The following MR imaging findings showed statistical significance $(p<0.05)$ : skip lesions, solitary lesion, intraspinal lesions, concentric collapse, abscess formation (para spinal, intraosseous and epidural), syrinx formation and
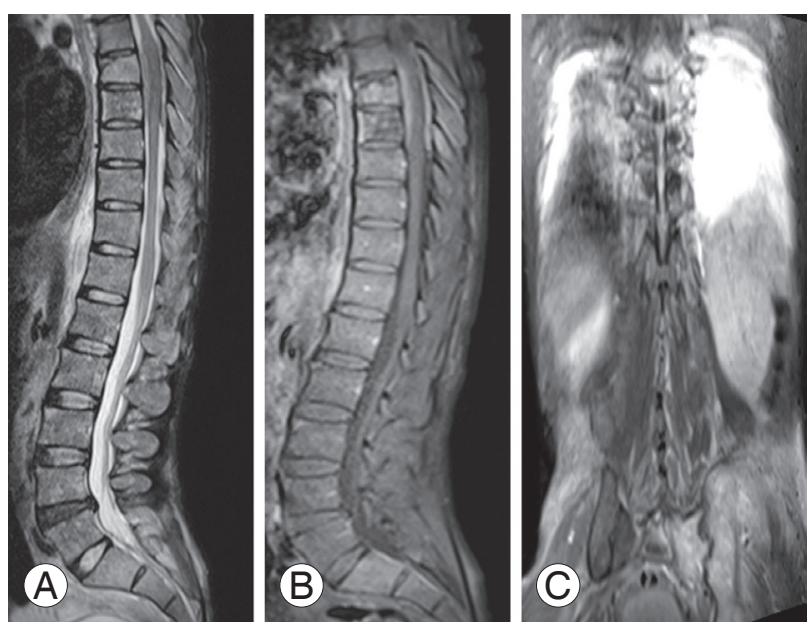

Fig. 9. Sagittal short tau inversion recovery (A) shows hyper-intensity involving the D7 and D8 vertebral body and ventral and dorsal epidural space causing thecal sac compression, which on post contrast T1-weighted fat-saturated image showed heterogeneous pattern of enhancement. Post contrast T1-weighted coronal fat-saturated image (C) shows hyper-intensity in left sacro-iliac joint with bilateral pleural effusion. The cytology findings revealed granuloma formation. site of distant focus other than the spine. Along with notable involvement of the site of skip lesions, these features help in differentiating these two entities and they have been summarized in Table 3 .

\section{Discussion}

According to the World Health Organization, tuberculosis accounts for most deaths in the world [1]. About one-third of the world population is infected with tuberculosis, which is quite high for a given disease. The exact incidence and prevalence of spinal tuberculosis in most parts of the world are not known. Approximately $10 \%$ of patients with extra-pulmonary tuberculosis have skeletal involvement, and the developing countries account for majority of spinal tuberculosis cases. The spine constitutes the most common site of involvement of skeletal tuberculosis, accounting for almost $50 \%$ of skeletal tuberculosis cases, followed by the hip and knee [1].

These numbers gives an incidence of between $1 \%$ and $2 \%$ for osteo-articular tuberculosis and $0.5 \%-1 \%$ for spinal tuberculosis [2]. Tuberculosis presents with multitude of clinical manifestations and alterations, thereby known as a great masquerader of diseases in different medical fields. This makes the characteristic features of the disease problematic, with most reports and articles describing the typical clinical and radiological picture, but followed by many atypical derivatives. As Pott's disease, also known
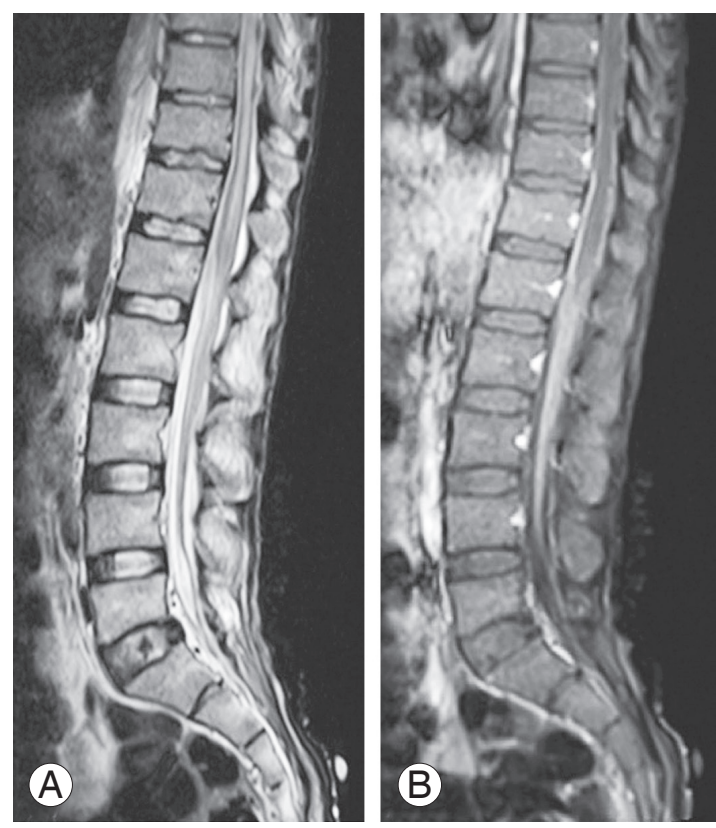

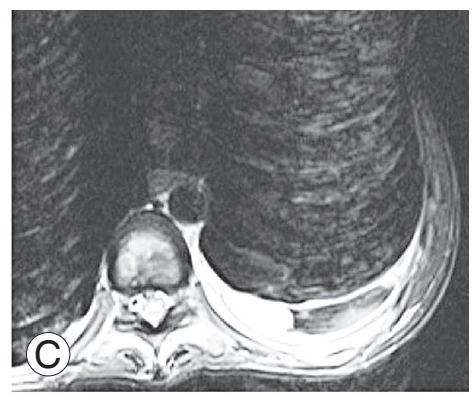

Fig. 10. Sagittal T2-weighted (A) image shows syrinx involving the dorso-lumbar spinal cord. Vertebras appeared normal. On post contrast T1-weighted sagittal image (B), there was leptomeningeal enhancement noticed along the dorsolumbar spine. Axial T2-weighted (C) image showed left sided pleural effusion. Cerebrospinal fluid analysis revealed increased lymphocytes with positive staining for acid-fast bacillus. 
Table 3. Comparison of specific features on MRI for differentiation between metastasis and atypical spinal tuberculosis

\begin{tabular}{|c|c|c|c|}
\hline MR Findings & Metastasis $(\mathrm{n}=20)$ & Tubercular spine $(\mathrm{n}=20)$ & $p$-value ${ }^{\mathrm{al}}$ \\
\hline Most common site (thoracic region) & $85(17 / 20)$ & $65(13 / 20)$ & 0.144 \\
\hline Cervical & $5(1)$ & $10(2)$ & \\
\hline Upper Thoracic & $20(4)$ & $15(3)$ & \\
\hline Lower Thoracic & $65(13)$ & $50(10)$ & \\
\hline Lumbar & $5(1)$ & $15(3)$ & \\
\hline Sacrum/coccyx & $5(1)$ & $10(2)$ & \\
\hline No. of skip lesions & $65(13 / 20)$ & $20(4 / 20)$ & 0.003 \\
\hline Site of skip lesions & & & - \\
\hline Cervical & 1 & 2 & \\
\hline Cervico-thoracic & 2 & 1 & \\
\hline Thoracic & 10 & 6 & \\
\hline Thoraco-lumbar & 4 & 4 & \\
\hline Lumbar & 1 & 3 & \\
\hline Lumbosacral & 1 & 2 & \\
\hline Sacrum/coccyx & 1 & 2 & \\
\hline \multicolumn{4}{|l|}{ Nature of lesions } \\
\hline Intra-spinal lesions & $20(4 / 20)$ & $60(12 / 20)$ & 0.009 \\
\hline Concentric collapse type & $60(12 / 20)$ & $10(2 / 20)$ & $<0.001$ \\
\hline Solitary lesion & $35(7 / 20)$ & $5(1 / 20)$ & 0.017 \\
\hline Meningeal Enhancement & $20(4 / 20)$ & $45(9 / 20)$ & 0.091 \\
\hline Isolated posterior elements involvement & $20(4 / 20)$ & $5(1 / 20)$ & 0.151 \\
\hline Isolated anterior element involvement & $55(11 / 20)$ & $65(13 / 20)$ & 0.520 \\
\hline Combined elements involvement & $75(15 / 20)$ & $70(14 / 20)$ & 0.723 \\
\hline \multicolumn{4}{|l|}{ Abscess } \\
\hline Para-spinal abscess & $0(0 / 20)$ & $30(6 / 20)$ & 0.007 \\
\hline Intra-osseous abscess & $0(0 / 20)$ & $25(5 / 20)$ & 0.016 \\
\hline Epidural abscess & $0(0 / 20)$ & $55(11 / 20)$ & $<0.001$ \\
\hline Syrinx formation & $10(2 / 20)$ & $40(8 / 20)$ & 0.030 \\
\hline Site of distant focus other than spine & $60(12 / 20)$ & $25(5 / 20)$ & 0.025 \\
\hline
\end{tabular}

Values are presented as \% (no. of cases).

$\mathrm{MRI}$, magnetic resonance image.

${ }^{\text {a) }}$ Chi-square test.

as the tuberculous spondylitis, frequently involves multiple contiguous spinal vertebrae, non-contiguous or skip pattern involvement with remote involvement have been reported in few cases [1,3-6].

Haematogenous dissemination is the most common mode of $M$. tuberculosis with entry into the dense vasculature of the vertebral bodies. Spinal tuberculosis occurs secondary to the primary infection site, which is either a pulmonary lesion or an infection of the genitourinary system [7-9], and spreading occurs either via the arterial or the venous route. In the sub-chondral region of each vertebra, an arterial arcade is derived from anterior and posterior spinal arteries and forms a rich vascular plexus. This vascular plexus facilitates haematogenous spread of the infection in the para-diskal regions. For paravertebral venous plexus (also known as Batson's plexus) in the vertebra, it is a valve-less system that allows free flow of blood in both directions, depending upon the pressure generated by the intra-abdominal and intra-thoracic cavities following strenuous activities such as coughing. In 
patients with non-contiguous vertebral tuberculosis, it is mainly the vertebral venous system that spreads the infection to multiple vertebrae.

The most common site of tuberculosis is the anterior inferior portion of the vertebral body. After that, it spreads into the central part of the body or disk. Three most common patterns of tuberculosis are para-diskal, anterior and central lesions of which para-diskal is the most common variety. In the central lesion, there is a collapse of the vertebral body with sparing of intervertebral disc, thereby leading to vertebra plana. In younger patients, the disk is primarily involved, because it is more vascularized. For older individuals, the disk is relatively spared, because of its age-related avascularity. Spread of the disease beneath the anterior or posterior longitudinal ligaments involves multiple contiguous vertebrae.

A feature most typically distinguishing spinal tuberculosis from pyogenic osteomyelitis is the relative sparing of the intervertebral discs. This is attributed to because of lack of proteolytic enzymes, which is responsible for the relative sparing of the intervertebral disc [10]. The discs are avascular in nature and that prevents them in becoming a seeding site for primary infection and the destruction of intervertebral disc begins only when the two adjacent vertebral bodies are compromised, leading to loss of its nutritional requirement.

Pott's disease has the tendency to form characteristic paravertebral or para-spinal abscesses. The extent of these collections usually exceeds the area of osseous involvement. Abscess formation can be seen in prevertebral, paravertebral, intra-osseous and epidural space. The wall of the abscess is characteristically thick and irregularly enhancing on both computed tomography and MR images.

A spinal lesion in a multiple metastatic form from systemic malignancy is the major entity from which multiple spinal tuberculosis must be distinguished. Spinal metastasis characteristically spares the intervertebral disc space, as does the anterior and central type of tuberculosis. When the spinal tuberculosis shows non-contiguous pattern of vertebral involvement, its imaging appearance can be mistaken for a metastatic malignancy. There are various factors that help in differentiating spinal tuberculosis from metastasis and these include the presence of paravertebral abscesses and a sub-ligamentous spread [11]. Lytic metastases were seen in metastasis from lung, breast and glottic primary tumours. Blastic metastases were mainly seen from breast and prostate cancers.
"Spinal metastases" is a vague term, which can be variably taken to refer to metastatic disease for any of the following (\% of the total): (1) Vertebral metastases (94\%): may have epidural extension. (2) Intradural extramedullary metastases (5\%). (3) Intramedually metastases (1\%).

\section{Epidemiology}

Approximately $90 \%$ of all spinal neoplasms (cord+ vertebral) are considered metastatic in origin. The presentation can be highly variable which includes sudden onset of the Brown-Sequard syndrome in patients with known metastatic cancer.

\section{Pathology}

Spread of malignant cells to the region is variable and includes: (1) Haematogenic: arterial or venous (via Batson's plexus). (2) Direct invasion: typically of para-spinal, retroperitoneal of pulmonary malignancies (3) Lymphatic: along the root sleeves. (4) Subarachnoid: intracanalicular seeding of primary and secondary central nervous system neoplasms.

As the vast majority of metastases to the region are to bone, this is reflected in the primaries which are encountered. It should be noted that breast and lung cancer and also melanoma are also the relatively common sources of intradural disease. Primaries include (\% of the total): breast $(22 \%)$, lung $(15 \%)$, prostate $(10 \%)$, lymphoma (10\%), etc.

Leptomeningeal metastases, also know as carcinomatous meningitis, refer to the spread of malignant cells through the CSF space. These cells can originate both from primary CNS tumours (e.g., drop-metastases), as well as from distant tumours that have metastasized (haematogenous spread). Clinical presentation is varied, but most commonly includes a headache, spine or radicular limb pain or sensory abnormalities, nausea and vomiting, and focal neurological deficits. Signs of meningitis are present only in a minority of cases. Various tumours responsible for leptomeningeal metastasis includes glioblastoma, ependymoma etc., as well as from haematogenous dissemination of primary malignancies, which includes breast, lung, prostate cancer and leukaemias etc. On MRI, these lesions show leptomeningeal enhancement scattered over in a 'sugar coated' manner.

Non-contiguous pattern of vertebral involvement in 
spinal tuberculosis is far more common (16.3\%) than that reported in literature [1]. There was a trend towards a higher incidence of neurological involvement in the noncontiguous group compared to the single focus group [1]. This trend may not necessarily reflect a more severe disease, but instead may be a product of heightened probability due to the increased levels involved and/or the "double-crush" neural phenomenon. Non-contiguous (atypical) spinal tuberculosis is not an obvious manifestation of HIV, multi-drug resistant tuberculosis or chronicity as in our patients. It is not considered a "fulminant" type of tuberculosis as demonstrated by Emel et al. [3].

Naim-Ur-Rahman et al. [12] reported various features of atypical osseous tuberculosis lesions of the spine [13], where firstly and foremost, atypical vertebral lesions readily involve the neural arch, sparing the vertebral body and discs (also known as "posterior Pott's spine"). Secondly, the skip lesions are often present, which are defined as the involvement of two or more vertebral lesions separated by an uninvolved vertebra. Finally, these lesions may manifest in the form of solid tumours with no evidence of any abscess formation and therefore, are almost indistinguishable from a tumour.

In our study, the findings like lung consolidation, pleural empyema, sacroilitis and communicating hydrocephalus were noted in patients with tuberculosis. No association of these findings were noted for metastasis.

The current study showed various imaging features for differentiating between atypical spinal tuberculosis and spinal metastasis; however, only few showed statistical significance $(p<0.05)$ which included skip lesions, the site for skip lesions, solitary lesion, intra-spinal lesions, concentric collapse, abscess formation (para-spinal, intraosseous and epidural), syrinx formation and site of distant focus other than spine. Abscess formation was a notable feature for distinguishing a metastatic lesion as these rarely associated with a significant soft tissue component. Again the skip lesion and the site (thoracic) showed a predilection for metastasis as there is few literature on a skip pattern for spinal tuberculosis. The leptomeningeal spread showed no statistical significance, but the pattern of enhancement i.e., smooth or nodular helps in differentiating benign from malignant aetiologies. The distant foci of involvement including liver, lung and nodes were seen mostly in cases of metastasis as a result of haematogenous dissemination. Tubercular aetiology less frequently shows this pattern of spread.
Tuberculosis should be considered in the differential diagnosis of various spinal lesions including metastasis, fungal spondylodiskitis, sarcoidosis and lymphoma, particularly in endemic countries. Because spinal tuberculosis is considered as one of the great mimickers of disease as it could present in a variety of typical and atypical patterns, so proper imaging must be done in order to facilitate appropriate treatment.

\section{Conclusions}

The differential diagnosis of tubercular spondylidiscitis includes metastasis, fungal spondylodiskitis, sarcoidosis and lymphoma, particularly in certain parts of the world. The spinal tuberculosis-Pott's spine-is the major cause of morbidity and mortality, particularly in developing nations like India, and therefore, clinic-radiological correlation is a necessity. Spinal tuberculosis can present in a variety of typical and atypical patterns, and is one of the great mimickers of disease. As such, a thorough imaging protocol and proper history could help in making accurate diagnosis and facilitate good treatment.

In endemic countries like India, for tuberculosis, always consider the possibility of tubercular infection, if there is a destructive soft tissue vertebral mass in known cases of malignancy. Imaging features help in guiding the proper differentiation between atypical spinal tuberculosis and metastasis.

\section{Conflict of Interest}

No potential conflict of interest relevant to this article was reported.

\section{References}

1. Polley P, Dunn R. Noncontiguous spinal tuberculosis: incidence and management. Eur Spine J 2009;18: 1096-101.

2. Naim-ur-Rahman. Atypical forms of spinal tuberculosis. J Bone Joint Surg Br 1980;62:162-5.

3. Emel E, Guzey FK, Guzey D, Bas NS, Sel B, Alatas I. Non-contiguous multifocal spinal tuberculosis involving cervical, thoracic, lumbar and sacral segments: a case report. Eur Spine J 2006;15:1019-24.

4. Kulali A, Cobanoglu S, Ozyilmaz F. Spinal tuberculosis with circumferential involvement of two noncon- 
tiguous isolated vertebral levels: case report. Neurosurgery 1994;35:1154-8.

5. Janssens JP, de Haller R. Spinal tuberculosis in a developed country: a review of 26 cases with special emphasis on abscesses and neurologic complications. Clin Orthop Relat Res 1990;(257):67-75.

6. Turgut M. Spinal tuberculosis (Pott's disease): its clinical presentation, surgical management, and outcome: a survey study on 694 patients. Neurosurg Rev 2001;24:8-13.

7. Tuli SM. Tuberculosis of the skeletal system: bones, joints, spine, and bursal sheaths. New Delhi: Jaypee Brothers Medical Publishers; 1993.

8. Boachie-Adjei O, Squillante RG. Tuberculosis of the spine. Orthop Clin North Am 1996;27:95-103.
9. Schirmer P, Renault CA, Holodniy M. Is spinal tuberculosis contagious? Int J Infect Dis 2010;14:e659-66.

10. Chapman M, Murray RO, Stoker DJ. Tuberculosis of the bones and joints. Semin Roentgenol 1979;14:26682.

11. Joseffer SS, Cooper PR. Modern imaging of spinal tuberculosis. J Neurosurg Spine 2005;2:145-50.

12. Naim-Ur-Rahman, El-Bakry A, Jamjoom A, Jamjoom ZA, Kolawole TM. Atypical forms of spinal tuberculosis: case report and review of the literature. Surg Neurol 1999;51:602-7.

13. Evangelista E, Itti E, Malek Z, et al. Diagnostic value of $99 \mathrm{mTc}$-HMDP bone scan in atypical osseous tuberculosis mimicking multiple secondary metastases. Spine (Phila Pa 1976) 2004;29:E85-7. 